\title{
Frontières
}

\section{À la fin d'une longue, très longue journée}

\section{Éric Volant}

Volume 18, numéro 2, printemps 2006

La mort dans tous ses états

URI : https://id.erudit.org/iderudit/1073220ar

DOI : https://doi.org/10.7202/1073220ar

Aller au sommaire du numéro

Éditeur(s)

Université du Québec à Montréal

ISSN

1180-3479 (imprimé)

1916-0976 (numérique)

Découvrir la revue

\section{Citer cet article}

Volant, É. (2006). À la fin d’une longue, très longue journée. Frontières, 18(2), 41-45. https://doi.org/10.7202/1073220ar

\section{Résumé de l'article}

Notre propos est de donner brièvement les résultats d'une étude comparative des taux de suicide chez les personnes âgées selon les pays, les âges et le sexe. Ensuite, dans notre effort de comprendre la signification sociale de ces chiffres, nous nous inspirons de la thèse d'Émile Durkheim selon laquelle le phénomène du suicide est lié à la capacité plus ou moins grande d'une société d’intégrer certains groupes, comme les aînés. Enfin, nous explorerons les plages d'exclusion ou plutôt d'incapacité d'intégration des aînés, susceptibles de conduire à des comportements suicidaires. En bout de ligne, nous suggérerons un accompagnement discret des aînés, respectueux de leur libre vouloir de vivre.
Ce document est protégé par la loi sur le droit d'auteur. L'utilisation des services d'Érudit (y compris la reproduction) est assujettie à sa politique d'utilisation que vous pouvez consulter en ligne.

https://apropos.erudit.org/fr/usagers/politique-dutilisation/ 


\section{Résumé}

Notre propos est de donner brièvement les résultats d'une étude comparative des taux de suicide chez les personnes âgées selon les pays, les âges et le sexe. Ensuite, dans notre effort de comprendre la signification sociale de ces chiffres, nous nous inspirons de la thèse d'Émile Durkheim selon laquelle le phénomène du suicide est lié à la capacité plus ou moins grande d'une société d'intégrer certains groupes, comme les aînés. Enfin, nous explorerons les plages d'exclusion ou plutôt d'incapacité d'intégration des aînés, susceptibles de conduire à des comportements suicidaires. En bout de ligne, nous suggérerons un accompagnement discret des aînés, respectueux de leur libre vouloir de vivre.

Mots clés: taux de suicide - aînés intégration - accompagnement.

\section{Abstract}

This article briefly gives the results of a comparative study about the suicide rates of elderly people, according to countries, ages and sex. In our effort to understand the social meaning of these figures, we are inspired by Émile Durkheim's thesis, according to which the phenomenon of the suicide is connected to the capacity of a society to integrate certain groups, as the elder. Finally, we investigate how exclusion or rather incapacity of integration of elder is susceptible to lead to suicidal behavior. In end, we shall suggest a respectful and discreet accompanying of the elder in their choices.

Keywords: suicide rates - elder integration - accompanying.

\section{À la fin \\ d'une longue, très longue journée}

\section{Éric Volant, Ph. D., professeur associé, Département de sciences des religions, UQAM.}

À l'approche de la mort du vieux couple Nicolas et Pernelle, Albus Dumbledore confie à Harry Potter que, pour eux, la mort, "c'est comme se coucher à la fin d'une longue, très longue journée. Après tout, pour un esprit équilibré, la mort n'est qu'une grande aventure de plus» (Rowling, 2000, p. 290). Le lecteur ne sait pas ce que le grand sorcier entend par "esprit équilibré», mais il comprend que, au regard rétrospectif des personnes âgées, la vie paraît comme une seule journée dont certaines heures furent trop longues, pleines d'ennui ou de désarroi, et d'autres, trop brèves, plus denses ou intenses, plus joyeuses ou plus tragiques. Il n'est donc pas inusité de voir les vieux se préparer à la mort, petit à petit, comme à une dernière heure, une expérience ultime. En vieillissant, on se fait à l'idée de mourir. Non sans humour, Tristan Bernard raconte: «Mon père est mort, mon grand-père aussi.
Je crains que ce ne soit héréditaire.» Cependant, même si l'heure se fait proche, il ne faut pas en conclure qu'elle ne fait pas peur aux vieux, cette grande inconnue, car ils ne savent pas vraiment comment l'affronter. Elle nous réservera des surprises, cette heure qui précédera notre décès autant que l'autre qui la suivra. De quoi le lendemain de cette heure sera-t-il fait? De sommeil paisible ou de joyeux émerveillement? Ou sera-t-il néant? "Ce rien terrible et si tenace, cruel, ce rien sans fin instauré par la mort. [...] Mais comment se battre avec le vide?» (Germain, 1999, p. 82)

Rendu dans ma quatre-vingtième année, je me crois justifié de me compter parmi les vieillards dont il est question. Bien sûr, nous ne savons pas comment mourir, ni comment se passeront concrètement pour nous ces derniers instants. Jusqu'à quel degré serons-nous souffrants? Quelles pensées nous habiteront? Nous n'éprouvons sans doute plus des sentiments d'agressivité à l'égard de la mort, comme certains jeunes 
dont j'ai entendu crier la révolte contre une mort proche et cruelle, qui venait ravir leur jeune vie. La mort, nous ne la dénions plus, car nous la savons inéluctable. Nous ne sommes pas pour autant délivrés de la peur de la douleur qui l'accompagnera ou du regard, discret ou impudique, que les autres jetteront sur notre souffrance. Nous avons peur que la mort de notre conjoint, d'un de nos enfants ou d'un de nos amis nous rende trop fragiles pour en assumer le deuil. Par contre, n'ayez pas peur de nous parler de la mort ou de nous interroger sur elle, car cette question effleure notre pensée quotidiennement et ne nous surprend pas!

Il est important de saisir avec justesse l'attitude des vieillards face à leur propre mort, lorsqu'on aborde la question du suicide. Sans vouloir leur attribuer une pensée uniforme, ils y ont pensé bien avant vous comme une option possible et, somme toute, disponible. Si des personnes, parfois très âgées, se tuent et prennent pour arriver à leurs fins des moyens efficaces, c'est parce que leur décision de mourir est plus ferme que celle des jeunes gens ${ }^{1}$ dont les sentiments face au suicide semblent plus ambivalents (Charbonneau, 2002). Je ne suis pourtant pas si sûr, comme certains auteurs le pensent, qu'elles se tuent plus facilement parce que le sacrifice à faire serait plus léger à quatre-vingt-cinq ans qu'à soixante ans ou à quarante ans (Andrian, 1991). Par contre, je crois que leur suicide ne serait pas tant le détachement d'une vie dont elles ont pu pleinement apprécier le sens et la bonté, mais la cessation d'une journée qui n'en finit plus et dont l'ennui ou le tourment pèsent désormais trop lourds sur leurs épaules devenues fragiles. Cette toute première approche, sans doute trop impressionniste ou trop hâtive, de la question du suicide à partir de la perception partagée par les aînés de leur propre mort, mérite une confrontation avec la froide réalité des chiffres et avec leur plausible interprétation.

\section{LE POIDS DES NOMBRES ET LA FRAGILITÉ HUMAINE}

La présentation des données statistiques demeure une opération délicate, car derrière la froideur des chiffres anonymes se cachent des personnes qui ont éprouvé la souffrance et le désarroi. En consultant ces tableaux, des personnes en deuil d'un proche suicidé se sentent lésées dans leur mémoire vivante. Dans cette abstraction mathématique, elles ne reconnaissent pas la douleur concrète et déchirante du drame vécu par elles-mêmes et par leur parent, enfant ou ami suicidé. Se pose également la question de la fiabilité des statistiques. Avec raison, on soulève les problèmes de la sous-déclaration du suicide. En effet, à

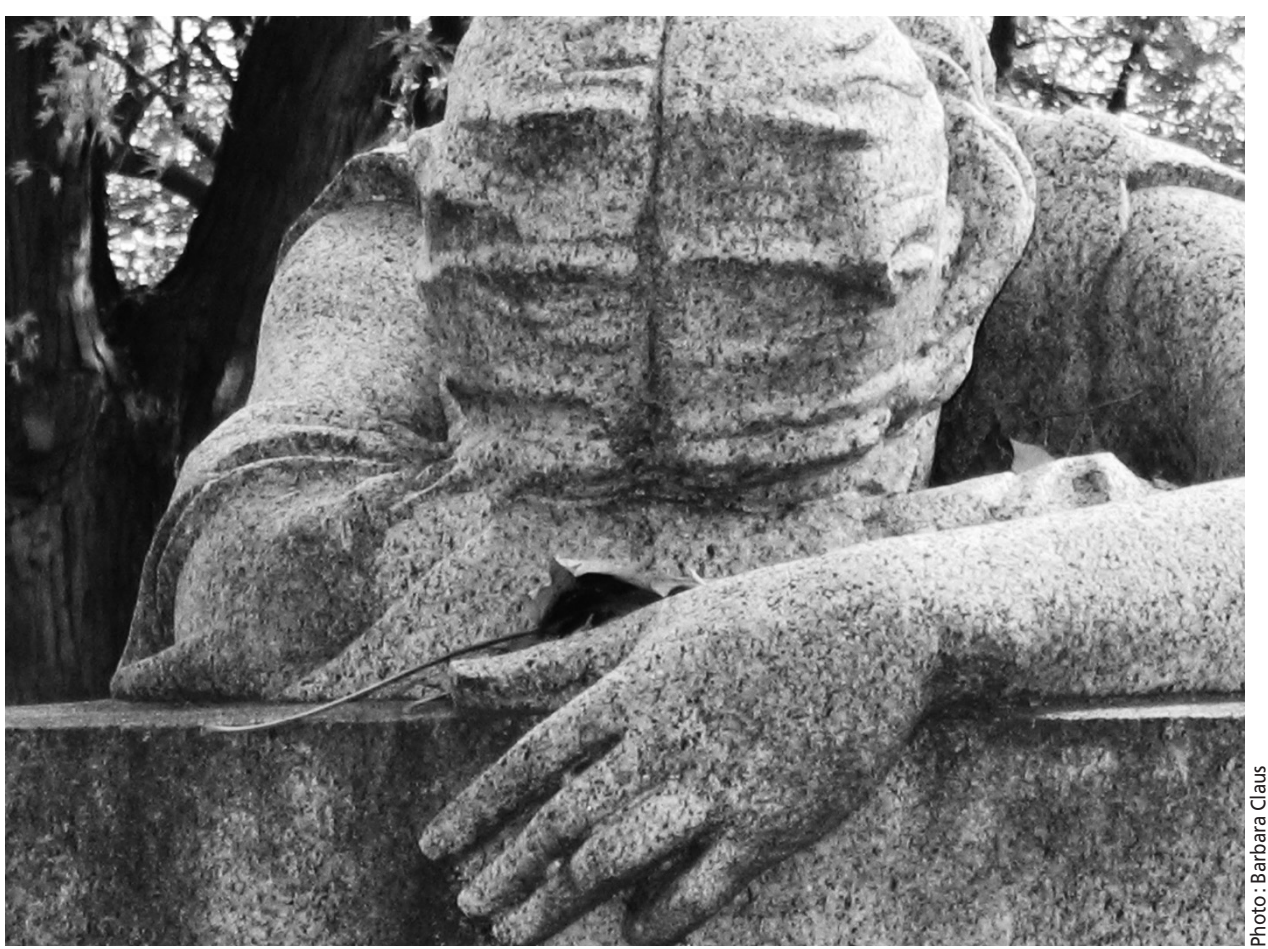

cause du tabou qui entoure une mort aussi dramatique que fatale, provoquant la réprobation sociale, il arrive que des suicides soient camouflés en morts accidentelles ou en accidents cardiovasculaires. En plus, on observe l'absence d'uniformité dans les critères d'attestation d'un décès par suicide selon les pays. Cependant, les statistiques officielles, celles de l'Organisation mondiale de la santé (OMS) ou celles des centres de recherche sur le suicide et de prévention de divers pays ou régions, notamment $\mathrm{du}$ Québec, tendent vers une validité suffisante ${ }^{2}$.

On se sert souvent des taux de suicide pour sonner la cloche d'alarme. La fable noire d'une humanité en désarroi! Effarant, le nombre de jeunes ou de vieux qui s'enlèvent la vie! Quatre ou huit personnes se tuent par jour! À chaque minute, il y a une ou des personnes qui...! Au point de vue clinique, on peut se poser d'ailleurs la question de la pertinence des taux de suicide pour la prévention et pour le traitement des personnes suicidaires. Dès sa fondation, la suicidologie s'est présentée comme une théorie sur la pratique clinique de la prévention du suicide. Elle s'est donc intéressée aux statistiques pour étudier la disponibilité des moyens dans une région donnée et surtout pour détecter la vulnérabilité de certains groupes d'âge ou de groupes sociaux face au suicide. Pour nous, les données statistiques sont utiles dans la mesure où elles nous aident à mieux comprendre le sens du suicide en tant que phénomène social et ses liens avec les conditions de vie et de santé, de paix et de guerre, avec les lois et les usages, la mentalité et la culture ou la mouvance et l'évolution d'une collectivité.
En ayant recours aux statistiques, Durkheim parvient à démontrer que les suicides sont reliés étroitement aux autres modes de conduite observables dans la collectivité. Ils ne constituent pas une «classe isolée de phénomènes monstrueux ", une déviance ou une pathologie, mais «une forme exagérée de pratiques usuelles» (Durkheim, 1981, p. 7). Toute l'argumentation de Durkheim, en faveur d'une analogie de structure entre le geste suicidaire et bien d'autres modes de conduite observables dans la collectivité, veut démontrer, en dernière analyse, que la subjectivité suicidaire n'est pas si éloignée ni si différente de la mentalité générale d'une société. Elle est un miroir dans lequel se reflètent les failles et les blessures de la tragédie collective. Cette vision du suicide, en tant que répercussion individualisée d'un phénomène social, est susceptible de transformer les modèles d'intervention auprès des personnes suicidaires.

Dans la plupart des pays industrialisés, le taux de suicide monte avec l'âge et atteint son sommet chez les personnes de 75 ans et plus, comme le tableau l'indique, par exemple pour la France (1999). On enregistre à peu près les mêmes chiffres en Belgique: 23,4 pour les $65-74$ et 39,7 pour les 75 ans et plus (pour 100000 personnes). Par contre, la moyenne en France pour tous les âges est de 17,5 tandis que, en Belgique elle s'élève à 21,1. Cette progression selon l'âge de la population se produit aussi en Allemagne, mais d'une façon encore plus graduelle: 7,7 (15-24), 10,6 (25-34), 14,7 (35-44), 17,5 (45-54), 18,1 (55-64), 19,1 (65-74), 31,1 (75 et plus). La moyenne de 
tous les groupes d'âges y est de 13,5, donc plus basse qu'en France et qu'en Belgique. En Suisse, le taux de suicide des 75 et plus est de 44,8, tandis que le taux de suicide de tous les âges confondus est de 19,1. L'Autriche affiche les mêmes chiffres 44,8 pour les 75 ans et plus tandis que la moyenne dans la population générale est de 19,3. Dans tous ces pays, les personnes de 75 ans et plus atteignent des taux de suicide plus élevés que ceux de la population nationale. En Hongrie, le taux des suicides des 75 ans et plus est de 62,9 tandis que la moyenne nationale est de 28,0. Les taux de suicide les plus élevés au monde sont enregistrés à Cuba, 68,0 (1996), et en Chine rurale, 117,6 (1999).

Aux États-Unis, le taux de suicide est considérablement plus bas que dans les pays mentionnés, tandis que les disparités entre les âges sont aussi moins prononcées. Toutefois, les personnes de 75 ans et plus ont un taux de suicide qui dépasse de loin le taux national. Au Canada, le taux de suicide des personnes âgées est moins élevé que celui des 15-24, des 25-34, des 35-44 et des 45-54. Il est plus bas que la moyenne nationale. Au Québec en 1999, ce sont également les jeunes qui se suicident plus que les personnes âgées: 21,0 (15 à 19 ans), 25,5 (20 à 24), 26,9 (25 à 29), 28,6 (30 à 34), 31,8 (35 à 39), 37,1 (40 à 44), 34,3 (45 à 49), 24,5 (50 à 54), 21,3 (55 à 59), 19,4 (60 à 64), 17,9 (65 à 69), 19,4 (70 à 74$), 16,1$ (75 à 80), 17,1 (80 à 84) et 20,4 (85 à 89). En revanche, les hommes de 65 à 69 ans $(33,0)$ et de 70 à $74(35,2)$ enregistrent des taux à peu près semblables à ceux des hommes de 15 à 20 ans $(34,0)$ et de 55 à 59 ans $(32,8)$. Les femmes de 70 ans et plus se suicident beaucoup moins que les femmes plus jeunes. Le taux le plus élevé chez les femmes est celui des 40-44 $(21,0)$ et des 45-49 $(16,6)$. Au Nunavut, en 2001, les taux sont extrêmement élevés: 80,2 pour l'ensemble de la population, soit 131,9 pour les hommes et 24,8 pour les femmes.

Dans la grande majorité des pays, les hommes se suicident beaucoup plus que les femmes, exception faite pour la Chine où l'on enregistre, par 100000 personnes, 13,0 de suicides d'hommes et 14,8 de suicides de femmes. En Chine rurale, ce sont surtout les jeunes femmes qui se suicident le plus. Chez les 25-34 ans: 20,2 hommes et 33,3 femmes; chez les 35-44 ans: 19,6 hommes et 26,5 femmes; chez les 45-54 ans: 28,9 hommes et 34,2 femmes; chez les 75 ans et plus, 139,7 hommes et 102,2 femmes.

Les hommes de 75 ans et plus constituent une tranche de la population extrêmement vulnérable au suicide. En France, ils se suicident quatre fois plus que les femmes $(80,5 / 17,5)$, en Lituanie trois fois plus $(84,9 / 28,2)$, en Hongrie quatre fois plus $(121,1 / 34,6)$, aux États-Unis $(42,4 / 4,0)$ et au Canada dix fois plus $(27,7 / 2,8)$. Au Québec, les hommes de 75-79 ans se suicident six fois plus que les femmes $(28,7 / 4,4)$, ceux de $80-84$ ans, cinq fois plus $(28,7 / 5,5)$. En 1997, les hommes de 85 ans et plus enregistrent un taux qui est presque le double du taux national des hommes $(29,1)$. En 1999, les hommes de 85 ans et plus se suicident cinq fois plus que les femmes $(33,8-7,1)$, alors que le taux national pour l'ensemble de la population est de 19,1 toujours par 100000 personnes $^{3}$. La différence dans le taux de suicides entre hommes et femmes d'âge avancé peut s'expliquer en partie par le fait que les femmes restent mieux intégrées dans leur réseau familial et assurent la continuité intergénérationnelle. En ce qui concerne les veufs, le décès de la conjointe donne à l'époux le sentiment d'avoir perdu non seulement une épouse, mais aussi une mère qui veillait sur sa santé et s'adonnait à la gestion des activités domestiques, surtout dans les couples où le partage des tâches est pour ainsi dire inexistant (Andrian, 1997, et Volant, 2001, p. 353-355).

\section{INTÉGRATION SOCIALE ET MISE À L'ÉCART}

L'exclusion est une catégorie utile à la compréhension sociale des données statistiques du suicide, notamment du suicide des personnes âgées. Encore qu'il convient de définir les termes. Qui sont les «personnes âgées »? Jusqu'aux années 1980 inclusivement, nous avons été habitués à considérer l'âge de la retraite (65 ans) comme le point de démarcation à partir duquel une personne est appelée "vieillard» ou «aîné». Depuis l'avènement des cohortes issues des conditions favorables à une retraite anticipée (60 ans) à la fin des années 1990 et grâce à l'amélioration des conditions de vie et de santé, les frontières ont été déplacées. Dès lors, on compte comme «vieillards» les personnes de 75 ans et plus et comme «grands vieillards» les 90 ans et plus. Et là encore, il faut nettement distinguer entre les personnes en bonne santé, toujours très actives socialement et intellectuellement, les personnes de plus faible santé physique ou mentale et les grands malades.

\section{LES TAUX* DE SUICIDE SELON L'ÂGE ET LE SEXE DANS QUATRE PAYS**}

\begin{tabular}{|c|c|c|c|c|c|c|c|c|c|c|}
\hline \multirow[b]{2}{*}{ PAYS } & \multicolumn{8}{|c|}{ Âges } & \multirow{2}{*}{$\begin{array}{l}\text { Tous les } \\
\text { groupes d'âges }\end{array}$} & \multirow{2}{*}{$\begin{array}{l}\text { Nombre } \\
\text { de suicides }\end{array}$} \\
\hline & $5-14$ & $15-24$ & 29-34 & $35-44$ & $45-54$ & $55-64$ & $65-74$ & $75+$ & & \\
\hline \multicolumn{11}{|l|}{ Canada (2000) } \\
\hline Taux global & 1,1 & 13,0 & 13,7 & 17,4 & 16,6 & 13,0 & 10,5 & 10,3 & 11,7 & 3605 \\
\hline Hommes & 1,4 & 20,2 & 21,7 & 27,4 & 24,0 & 20,6 & 16,9 & 27,7 & 18,4 & 2798 \\
\hline Femmes & 0,9 & 5,5 & 5,5 & 7,3 & 9,2 & 5,6 & 4,9 & 2,8 & 5,2 & 807 \\
\hline \multicolumn{11}{|c|}{ États-Unis (2000) } \\
\hline Taux global & 0,7 & 10,2 & 12,0 & 14,5 & 12,1 & 12,1 & 12,5 & 18,2 & 10,8 & 30622 \\
\hline Hommes & 1,2 & 17,0 & 19,6 & 22,8 & 22,4 & 19,4 & 22,7 & 42,4 & 17,6 & 24672 \\
\hline Femmes & 0,3 & 3,0 & 4,3 & 6,4 & 6,7 & 5,4 & 4,0 & 4,0 & 4,1 & 5950 \\
\hline \multicolumn{11}{|l|}{ France (1999) } \\
\hline Taux global & 0,4 & 7,9 & 16,9 & 23,4 & 24,6 & 22,5 & 25,9 & 39,7 & 17,5 & 10268 \\
\hline Hommes & 0,5 & 12,3 & 26,1 & 35,8 & 34,3 & 31,3 & 39,6 & 80,5 & 26,1 & 7427 \\
\hline Femmes & 0,2 & 3,4 & 7,7 & 11,2 & 14,9 & 14,1 & 14,8 & 17,5 & 9,4 & 2841 \\
\hline \multicolumn{11}{|c|}{ Hongrie (2002) } \\
\hline Taux global & 0,6 & 10,5 & 17,6 & 41,7 & 45,7 & 35,5 & 38,5 & 62,9 & 28,0 & 2843 \\
\hline Hommes & 0,7 & 17,0 & 29,5 & 69,9 & 74,0 & 60,5 & 73,9 & 121,1 & 45,5 & 2195 \\
\hline Femmes & 0,5 & 3,6 & 5,4 & 13,8 & 19,7 & 15,3 & 15,1 & 34,6 & 12,2 & 648 \\
\hline
\end{tabular}

* Taux par 100000 personnes.

** Source principale: Organisation mondiale de la santé (OMS). Taux par 100000 personnes. À partir des données de cet organisme et à partir de données provenant de centres de recherche sur le suicide et de centres de prévention du suicide, nous avons construit des tableaux de tous les pays de l'Europe, de l'Amérique du Nord, de l'Amérique centrale et de quelques pays d'Afrique, d'Asie et de l'Amérique du Sud. 
Qu'est-ce que l'exclusion? L'exclusion est un terme d'ordre spatial ( $e x=$ hors de et claudere $=$ fermer), qui évoque l'image d'un territoire clôturé avec défense d'accès. «Exclure», c'est donc déclarer légitime un espace social, en définir les limites et en écarter des individus ou des groupes jugés incompatibles avec les valeurs, estimées comme conditions d'appartenance à cet espace (Dhoquois, 1989, p. 12; Xiberras, 1994; Volant, 1996, p. 125-141).

En ce qui concerne le sort des personnes âgées au Québec, on n'observe pas d'exclusion au sens strict, exception faite de certains cas individuels "difficiles» à classifier ou à traiter selon les normes habituelles. En effet, il n'y a ni rejet structural ni interdiction d'accès aux services offerts par les institutions publiques, notamment dans les domaines de la santé et du bienêtre social. Les discours officiels témoignent plutôt du respect dû à l'égard des aînés, même si dans la pratique quotidienne, on peut constater des négligences, des erreurs, des maladresses et des mauvais traitements. Une certaine méconnaissance de la psychologie des aînés, de leurs besoins et de leurs attentes, dans l'approche clinique ou administrative de ces personnes, est un signe qui révèle une relative incapacité de la société d'intégrer les personnes âgées. C'est ici que la thèse de Durkheim prend toute sa force: «le suicide varie en raison inverse du degré d'intégration des groupes sociaux dont fait partie l'individu» (Durkheim, 1981, p. 223). Le groupe social des aînés se montre vulnérable à des comportements suicidaires dans la mesure où il est trop peu, ou parfois trop fortement, intégré.

Dans le domaine familial, le deuil du conjoint, d'un enfant, d'un petit-enfant ou d'un proche bien-aimé, la séparation ou le divorce et surtout la violence familiale constituent, pour les personnes âgées, des moments très critiques. Il y a parfois de ces situations dramatiques qui passent inaperçues. Un vieux frappe encore sa vieille qui, ne sachant quoi faire, fait parfois des fugues qui sont interprétées par leurs enfants adultes comme des «absences d'esprit». Ou les fugues sont-elles des petites folies qui dérangent le vieux et le mettent en colère? Au plan social, les mauvaises conditions d'hébergement, l'insuffisance des traitements médicaux, le non-respect de la capacité de consentement (ou de refus) aux dits traitements, le préjugé de l'inutilité sociale ou économique des personnes âgées, perceptible dans l'attitude et le comportement des acteurs sociaux ou des citoyens, font que la vieillesse est subie par certaines d'entre elles comme une sanction ou comme une punition injuste. Des cas d'intégration excessive, comme celui d'un contrôle excessif des médicaments ou de l'alimentation,

LES PERSONNES ÂGÉES NE VEULENT PAS ÊTRE TRAITÉES

COMME DES ENFANTS OU DES IRRESPONSABLES.

chez des personnes vaillantes et lucides, ou, dans certaines maisons d'hébergement, une surveillance excessive de leurs allées et venues ou de leurs visites les insultent ou les blessent. Les personnes âgées ne veulent pas être traitées comme des enfants ou des irresponsables, elles qui ont eu un passé de responsabilités diverses et ont encore un esprit très éveillé, même si leur démarche est moins vive et le débit de leurs paroles plus lent ou plus hésitant.

Une contrainte répressive, accompagnée de la conscience aiguë de l'irréversibilité de leur processus de vieillissement, peut mener à la dépression ou au désespoir. Le suicide leur apparaît comme une issue légitime à une mise à l'écart fondée dans le réel et fortement ressentie. Des vieux ou des vieilles deviennent "hors d'ordre», même si, selon leur rythme et leur personnalité, leurs facultés et leurs goûts, ils sont encore capables de contribuer à l'orientation de leur collectivité. Le fait que la personne âgée souffre de maladie chronique ou d'un handicap physique, qu'elle vit dans la pauvreté et l'isolement, qu'elle ne produit plus directement et coûte cher en dépenses publiques porte la société à estimer plus facilement «acceptable» le suicide d'une personne âgée. Si celle-ci entre dans ce jeu cynique d'argumentation socioéconomique, elle deviendra, par intégration excessive, la victime émissaire d'une mort volontaire, sans risque et bénéfique à la survie de la communauté.

\section{ACCUMULATION DES PERTES ET DÉSENGAGEMENT}

Les pertes multiples qui, accumulées, deviennent une plaie béante, préfigurent la béance de la mort. Elles créent, chez des personnes âgées, une brèche où peut s'infiltrer le désir d'en finir avec la vie. Voici un échantillon des pertes qui peuvent se concentrer sur une seule personne: la perte de sa santé et de son intégrité physique; la diminution auditive et visuelle avec limitation des déplacements et confinement à la chambre; la perte par la mort d'un conjoint, d'un enfant ou d'un proche; la perte de son domicile, de son environnement habituel, de son jardin, de ses objets familiers; la perte des enfants par leur éloignement ou leur indifférence qui se manifeste par la réduction ou l'absence des visites; la perte de relations, car les gens de son âge meurent, sont malades et ne sortent plus; le manque de sorties et d'activités sociales; le souvenir d'une perte très douloureuse qui refait surface avec une grande intensité, par exemple, un enfant de dix ans mortellement happé par une auto; la perte de son innocence ou le regret déchirant des erreurs ou des omissions de son passé, le sentiment de la médiocrité de sa vie et de sa personnalité, de l'insignifiance de sa vie présente, le manque de confiance en soi portant à fuir le contact avec les autres qui, par pudeur ou discrétion, s'éloignent à leur tour.

Toutes ces pertes contribuent à un rétrécissement de la vie et atteignent leur paroxysme dans la réduction du pouvoir d'agir. La perte du contrôle de la situation, l'incapacité ou la peur de ne plus être capable d'assumer, de façon autonome, les actes de la vie quotidienne ou de dépendre de l'aide d'autrui, l'impuissance d'agir et la souffrance causée par cette impuissance peuvent conduire à une diminution de l'effort pour exister (Ricoeur, 1995, p. 106). La dépendance à l'alcool ou aux médicaments sont des signes d'une dépression qui, elle, fait suite à la mise à l'écart et à la brisure $\mathrm{du}$ lien social qui trouvent leur origine dans ce sentiment d'impuissance d'agir. S'il n'y a plus rien à attendre de la vie, la moindre douleur devient plus difficile à supporter. Il convient de se convaincre d'une évidence. Un homme, une femme de 85 ans peut souffrir autant qu'à vingt ou qu'à quarante ans, même si elle reste muette. Le silence d'un souffrant demeure difficile à interpréter. Il y a danger de sous-estimer ou de surestimer la douleur ${ }^{4}$. On appelle «syndrome de glissement » les signaux qui révèlent le désengagement de certains vieillards devant la vie: le refus de se lever ou de sortir, de se laver et de s'alimenter, de recevoir des soins médicaux et de communiquer. Comme le note Almerindo Lessa, le suicide est «une recherche d'échec à la mort», car «on tue la mort en se tuant soi-même». Comme le formule plaisamment Jacques Prévert, «À la douane de la mort, ils ne déclarent même plus la guerre, ils la passent en fraude» (Le Guen, 1992). Le suicide se présente alors comme une «fuite anticipée» par peur de mourir vivant d'une longue dégradation (Althusser, 1994; Pommier, 1998).

\section{ENTRE LE DÉFAITISME ET LA TOUTE-PUISSANCE}

La tâche des intervenants est fort délicate. On ne s'improvise pas intervenant auprès des vieux, même si on est vieux soimême et non plus si l'on est jeune et bardé de diplômes. Des interventions justifiées techniquement ne sont pas nécessairement 
opportunes, si elles ne tiennent pas compte des vrais besoins et de la volonté réelle de la personne âgée, non pas en général, mais de cette personne-ci en particulier. Non pas la volonté, telle qu'elle est interprétée par l'intervenant qui applique à tout le monde la même consigne astucieuse: "il ne veut pas mourir, mais vivre autrement». Or, on ne peut pas vouloir sauver coûte que coûte la vie de l'autre, car on ne peut pas tout faire. En revanche, ne rien faire, "parce que c'est inutile», par une sorte de fatalisme n'est pas mieux, car on ne peut pas abandonner une personne. Toute personne, jeune ou âgée, a besoin d'un accompagnement discret et empathique, capable de discerner le degré d'intensité de son désir d'une présence attentive et d'un soutien effectif pour vivre ainsi que de son désir de désengagement par rapport à la vie (Le Guen, 1990). Parce que son travail de deuil a déjà commencé, elle envisage peut-être sa mort avec détachement et lucidité, sans nécessairement recourir au suicide.

\section{Bibliographie}

ALTHUSSER, L. (1994). L'avenir dure longtemps, Paris, Gallimard, "Livre de poche».

ANDRIAN, J. (1991). «Être vieux», Mutations, $\mathrm{n}^{\circ} 124$.

ANDRIAN, J. (1997). «Suicide au grand âge », Thanatologie, $\mathrm{n}^{\text {os }}$ 109-110, p. 95-114.

CHARBONNEAU, L. (2002). "Le suicide chez les personnes âgées », Équilibre en têtes, vol. $13, \mathrm{n}^{\circ} 2$.

DHOQUOIS, R. (1989). Appartenance et exclusion, Montréal, Paris, L'Harmattan.

DURKHEIM, É. (1981). Le suicide. Étude de sociologie, Paris, Presses universitaires de France.

GERMAIN, S. (1999). Tobie des marais, Paris, Gallimard.

LE GUEN, J.P. (1990). «Maison de retraite: Lieu de réengagement», <www.vivre100ans.fr>.

LE GUEN, J. P. (1992). «Quand la nuit l'emporte sur le jour», o. c.

POMMIER, G. (1998), Louis du Néant: La mélancolie d'Althusser, Paris, Aubier, «Psychanalyse».

RICOEUR, P. (1995). Réflexion faite. Autobiographie intellectuelle, Paris, L'Esprit.

ROWLING, J. K. (2000). Harry Potter à l'école des sorciers, Paris, Gallimard.

VOLANT, É. (1996). "Le chômage d'exclusion ", Religiologiques, n ${ }^{\circ} 13$, p. 125-141.

VOLANT, É. (2001). Le dictionnaire des suicides, Montréal, Liber.

XIBERRAS, M. (1994). Les théories de l'exclusion, Paris, Méridiens Klincksieck.

\section{Notes}

1. Les jeunes se tuent facilement, parce qu'ils n'ont pas encore pu apprécier, à sa juste valeur, la riche densité de la vie ou encore par déni de la mort. La mort peut arriver à tous sauf à eux. La mort ne fait pas encore partie de l'univers de leur pensée. Cependant, des nuances et des distinctions s'imposent, à cause de la diversité de tempérament, de personnalité, de culture et d'éducation.

2. Au Québec, tous les cas de suicide, considéré comme mort violente, sont traités par le coroner. Or, «les coroners seraient moins enclins à ouvrir une enquête lorsqu'il s'agit de la mort d'un aîné malade. Sans trop de risque d'erreur, nous pouvons penser que les taux de suicide des aînés sont plus importants que ceux qui sont présentés officiellement». (I. Morin, «Un papi aussi, ça peut se suicider? », Vivre, vol. 3, n 4, février-mars 2004, p. 24-25.)

3. Selon une étude menée par des chercheurs du Centre de recherche de l'Institut universitaire de gériatrie sur le vieillissement sous la direction de Michel Préville de l'Université de Sherbrooke, au Québec, le taux de suicide des personnes de 65 ans et plus a augmenté de 85,4\% entre 1977 et 1999 . Cette croissance se serait produite malgré l'amélioration des conditions de vie des personnes âgées. Les données dont nous disposons ne révèlent pas de résultats aussi pessimistes. En plus, nous ne pouvons nous empêcher d'être un peu sceptique devant le désastre annoncé lorsque les experts estiment que le nombre de suicides augmentera de 248\% d'ici 2043.

4. Les travaux de Jean-Pierre Le Guen, directeur du Centre Saint-Exupéry à Brest sur le suicide des personnes âgées, compilés par Laure Lasfargues $<$ www.vivre100ans.fr $>$, ont guidé ma réflexion. 